\title{
Iron deficiency is not always innocent in childhood: a rare diagnosis of collagenous gastritis
}

\author{
Sevim Çakar ${ }^{1 \oplus}$, Yasemin Çakır ${ }^{2 \oplus}$ \\ Departments of ${ }^{1}$ Pediatric Gastroenterology and ${ }^{2}$ Pathology, University of Health Sciences, Dr. Behçet Uz Childrens Hospital, Izmir, \\ Turkey.
}

\begin{abstract}
Background. Collagenous gastritis (CG) is a very rare disease with still lots of unknowns, characterized by the subepithelial collagenous band in the gastric mucosa associated with a mixed inflammatory infiltrate in the lamina propria.

Case. Iron deficiency anemia is the most common and usually single laboratory finding without any complaint at the time of diagnosis. This entity should be well-known so that we can examine and refer the patient to a pediatric gastroenterologist for differential diagnosis.
\end{abstract}

Conclusions. The histopathological evaluation, albeit invasive, is essential to exclude this diagnosis. We present a 13-year-old girl with intractable iron deficiency anemia due to CG.

Key words: collagenous gastritis, iron deficiency, child, pathology, endoscopy.

Collagenous gastritis $(\mathrm{CG})$ is a rare disease especially in childhood that is characterized by the subepithelial deposition of collagen bands on histopathology in the gastric mucosa and still has unknown etiology. ${ }^{1}$ Iron deficiency anemia, abdominal pain and vomiting are common clinical presentations. The literature indicates that childhood-onset CG has a chronic disease course. ${ }^{2}$ This disease has been recognized in recent years and perhaps it is overlooked because it is not considered in more patients. Clinical diagnosis is yet impossible but endoscopic and histopathological evaluation is diagnostic and required.

\section{Case Report}

A 13-year-old girl was referred to our pediatric gastroenterology outpatient clinic for the evaluation of a two-year history of intractable

\footnotetext{
$\bowtie$ Sevim Çakar

drsevimgokgoz@gmail.com
}

Received 11th March 2021, revised 17th May 2021, accepted 17th May 2021. iron deficiency anemia. She had a diet rich in meat and meat products and a normal menstrual pattern. At the first visit at the age of 11, the patient did not have any gastrointestinal complaints such as vomiting, dysphagia, abdominal pain, diarrhea, melena.

On physical examination, the patient was obese with body weight in the 97th percentile, height in the 25-50th percentile, body mass index was 27 in the 97th percentile and there was minimal tenderness in her upper abdomen. Laboratory findings showed iron deficiency anemia with hemoglobin $(\mathrm{Hb})$ concentration of $8,6 \mathrm{~g} / \mathrm{dL}$, mean corpuscular volume (MCV) of $61 \mathrm{fl}$, serum iron level of $15 \mathrm{mg} / \mathrm{dL}$, serum ferritin of $1 \mathrm{ng} / \mathrm{mL}$ and total iron binding capacity (TIBC) of $415 \mathrm{mg} / \mathrm{dL}$ with normal infection parameters. Fecal occult blood test was negative. Anemia was treated with oral iron replacement therapy (4 mg/kg/d) for 6 months, but there was no response. Thus, parenteral iron replacement therapy had been administered and $\mathrm{Hb}$ level increased up to $12.5 \mathrm{~g} / \mathrm{dL}$.

At the age of 13 , her $\mathrm{Hb}$ level decreased to $9.7 \mathrm{~g} /$ $\mathrm{dL}$ and the patient was admitted to the pediatric 
gastroenterology department. Parasitic infections were excluded by performing repeated anal band tests and stool examinations. Helicobacter pylori infection was excluded by testing fecal Helicobacter pylori antigen and her celiac serology was negative.

In the upper gastrointestinal endoscopy for the evaluation of intractable iron deficiency anemia, there was no gastrointestinal bleeding, but endoscopic gastric images were grossly abnormal. The duodenum and esophagus were normal but the gastric corpus-fundus and antral mucosa was nodular. Gastric mucosal edema and erythema, as well as hyperplastic rugae were amongst the other macroscopical findings (Fig. 1). Biopsy specimens were routinely obtained from the duodenum, bulbus, esophagus, fundus, corpus and antrum. In the histopathological evaluation of all of the gastric mucosal specimens epithelial mucosa was normal but the subepithelial layer especially the lamina propria was characterized by the infiltration of chronic inflammatory cells and the deposition of collagen in focal areas. Multiple biopsies from the nodules showed patches of thick collagen bands, hyaline collagen deposition in the subepithelial layer strongly stained with Masson-trichrome and an increased number of plasma cells, eosinophils, and lymphocytes in the lamina propria. All of the gastric specimens revealed the same histopathologic changes (Fig. 2). Helicobacter pylori or granulomas were not detected in any of the samples. The patient received the diagnosis of CG by the pathological evaluation.

A high-dose proton pump inhibitor (PPI) with a dose of $2 \mathrm{mg} / \mathrm{kg} /$ day and oral iron supplementation was started. After the 6 weeks of treatment, her follow-up physical examination was completely normal but a control endoscopic evaluation was repeated because the $\mathrm{Hb}$ level rise was insufficient despite oral iron supplementation and PPI treatment. There was no tissue healing and similar histopathological findings persisted. Despite these findings the patient had no complaints and the physical examination was normal. Colonoscopy was not performed because the mucosal deposition of collagen has been shown usually to be limited to the stomach in childhood. In our case, there were no signs of colitis such as watery diarrhea and abdominal pain. We planned to perform a colonoscopy according to her clinical course to rule out collagenous colitis in case of lower
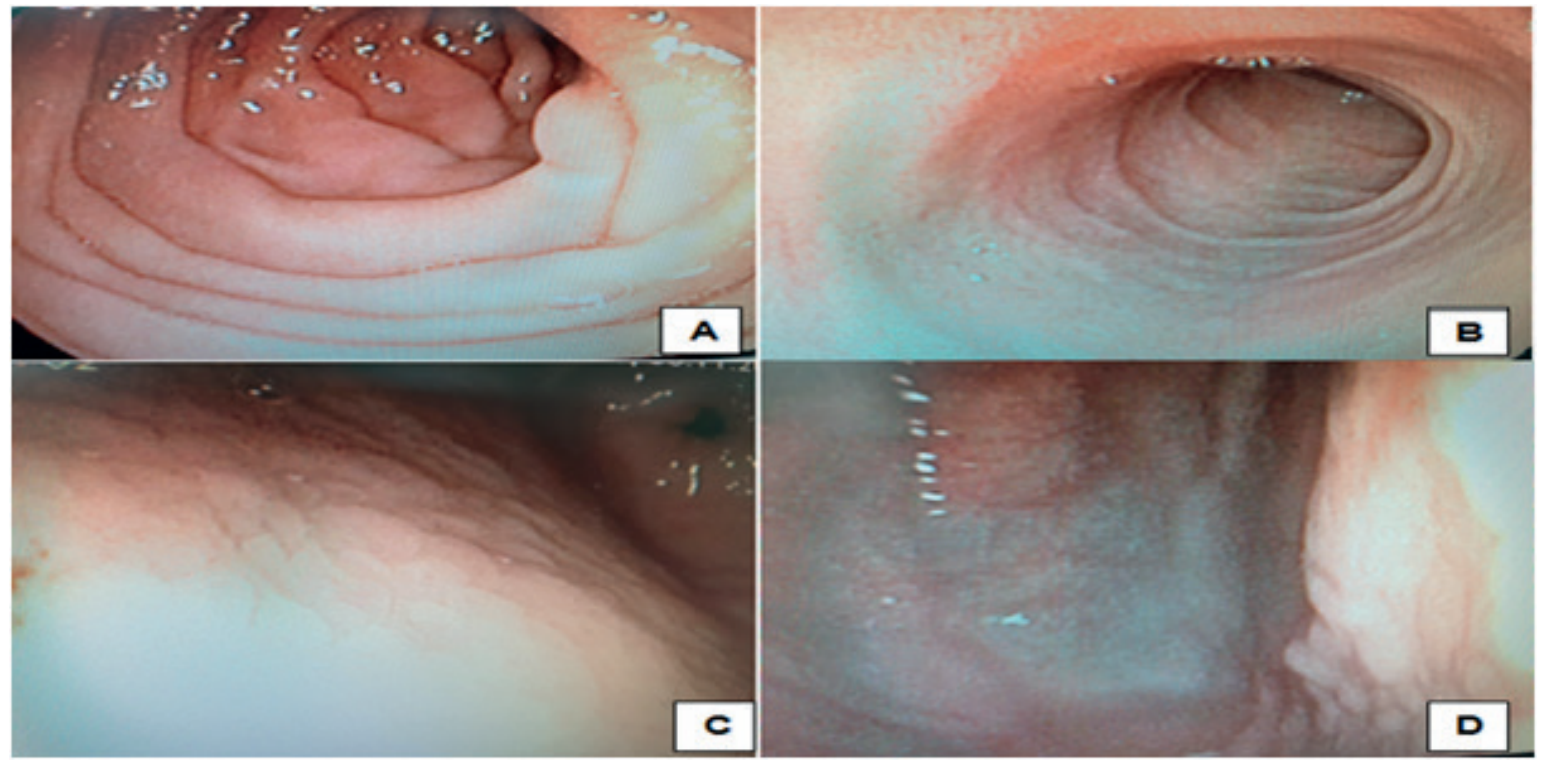

Fig. 1. Endoscopic findings of collagenous gastritis. A,B: duodenum and bulbus; completely normal evaluation. C,D: antrum and corpus; nodular lesions in the greater curvature of the gastric body. 

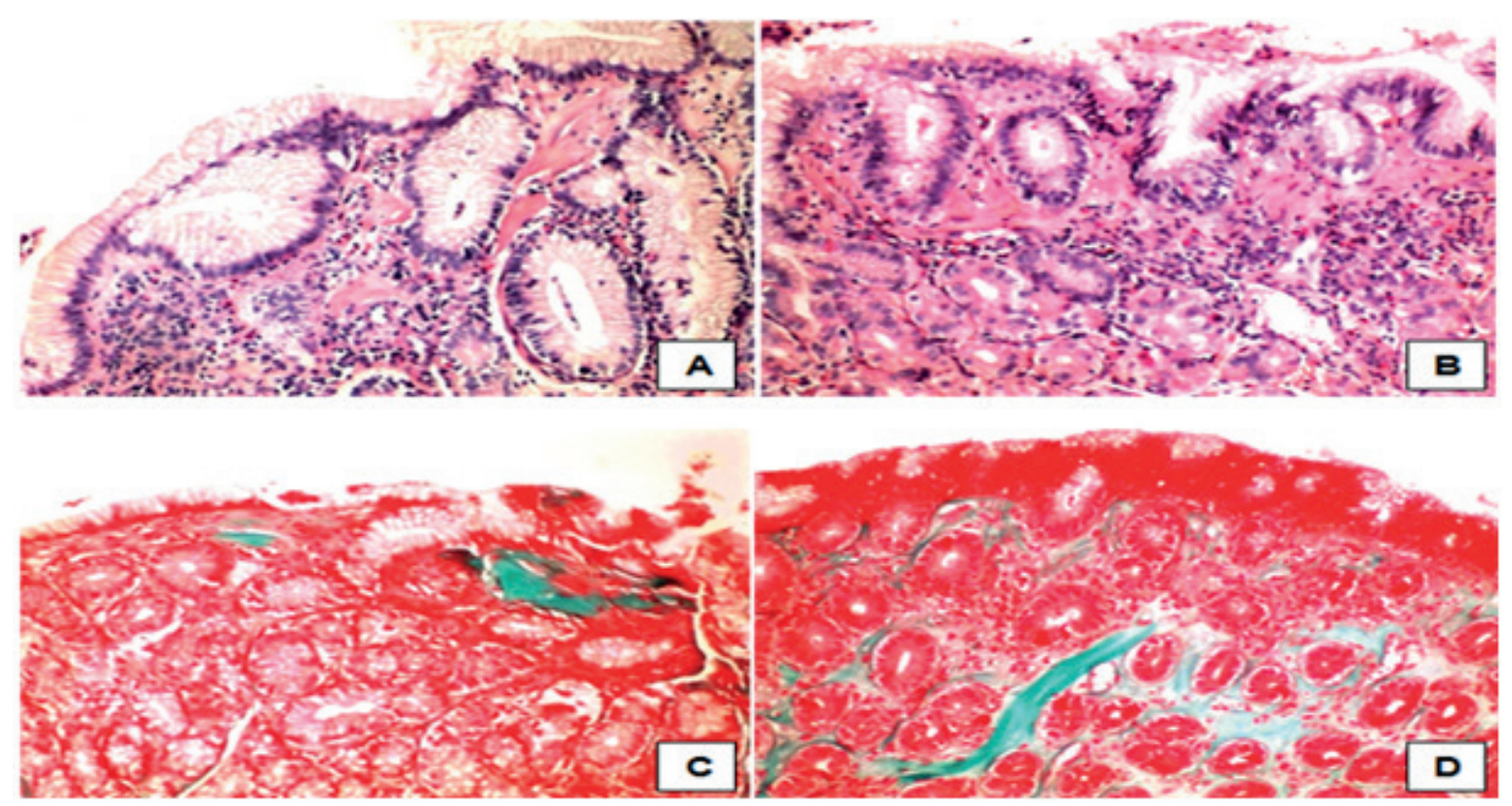

Fig. 2. Histological findings of collagenous gastritis. A,B: HE stain x 200, antrum and corpus ; thick collagenous subepithelial band. Inflammatory infiltrate in the lamina propria. C,D: Trichrome stain, antrum and corpus, thick subepith.

gastrointestinal symptoms. The follow-up was planned with intermittent iron treatment in our outpatient clinic.

The potential concomitant autoimmune diseases were excluded by the negative test results of Antinuclear antibody (ANA), Tissue transglutaminase immunoglobulin (Ig) A, Antithyroid peroxidase antibodies. Serum levels of amyloid A and Ig G, A, M were in normal range for her age.

Permission and informed consent was obtained from the patient and the family.

\section{Discussion}

Collagenous gastritis is a rare histopathological entity seen especially in children. Clinical diagnosis is impossible but histopathological evaluation is diagnostic and required. ${ }^{1}$ In cases in which dietary iron intake is adequate, occult blood loss cannot be demonstrated, infections (parasites, Helicobacter pylori...), malabsorption syndromes, allergic diseases are excluded, this disease should be included in the differential diagnosis and endoscopy should be performed when necessary. The first pediatric case was published in 1989 by Colletti and Trainer and female predominance was demonstrated in recent years. ${ }^{1-3}$

GG is characterized by the presence of a subepithelial collagen band (conventionally defined as being $>10 \mathrm{~mm}$ in thickness) in association with an inflammatory cell infiltrate in the lamina propria. ${ }^{4,5}$ It has been hypothesized that the deposition of collagen and protein exudate occurs due to an increase in vascular permeability but the pathophysiology is still unclear. $^{2}$ Iron deficiency is associated with chronic bleeding from dilated capillaries in the collagen layer. ${ }^{6}$ Instead of occult bleeding from the gastric mucosa, decreased iron absorption due to gastric hypochlorhydria can be another mechanism.

In the literature, two phenotypes of the disease have been described: a pediatric-onset and an adult-onset type. Recurrent abdominal pain and iron deficiency anemia, were the most common clinical presentations in pediatric-onset CG but upper gastrointestinal bleeding/hematemesis, 
recurrent vomiting and retrosternal pain were also reported..$^{7-9}$ In pediatric cases the collagenous mucosal inflammation is generally restricted to the stomach. All pediatric patients who presented with chronic diarrhea and malabsorption were found to have the adultonset phenotype characterized by concomitant collagenous gastritis and colitis. ${ }^{4,10-12}$ Our case presented with only anemia like many other reports in the literature with a prevalence of anemia up to $92 \%$ at presentation. ${ }^{7,8}$

The endoscopic image in all cases with CG is grossly abnormal like in ours. Features included nodularity of the gastric mucosa, mucosal edema and erythema, as well as hyperplastic rugae. By contrast, the patients with collagenous colitis had macroscopically normal colonoscopic images. Histopathologic findings of stomach biopsies show characteristic subepithelial hyaline deposits with an inflammatory infiltrate in the lamina propria of the affected areas. In previous histopathologic studies have reported the eosinophil-rich inflammatory infiltrate $(>30$ eosinophils/ high-power field) rate of $50 \%-62 \%$ in children with CG. The lymphocytic gastritis ( $>25$ surface intraepithelial lymphocytes/ 100 epithelial cells) like pattern was found in $<10 \%$ of the cases. ${ }^{8}$ Follow-up gastroscopies were performed in the largest pediatric cohort (15 patient) study and none of them had evidence of endoscopic or histologic improvement of the gastric mucosal pathology in all treatment regimens like our case. ${ }^{3}$ However, in the same study there was no worsening of the mucosal inflammation over time, and no patients developed intestinal metaplasia or advanced mucosal atrophy in the gastric corpus. Moreover, these patients had been evaluated with at least one colonoscopy with multiple mucosal biopsies during the follow-up period. Only one patient with associated collagenous colitis, no additional pathologies were noted in the colonic or ileal biopsies of the remaining patients.

Autoimmune diseases such as celiac disease, type 1 diabetes and psoriasis have been reported in children with $\mathrm{CG}^{7,8,13}$ In a study evaluating the frequency of heredity for autoimmune diseases in CG, the rate of positive autoantibodies was $40 \%$ but none of these patients had an autoimmune disease during the 11 years follow-up period. ${ }^{3}$ In our patient, the autoantibodies were also negative. CG and associated common variable immune deficiency and selective Ig A deficiency have previously been reported. ${ }^{8,14,15}$ In a case report of pediatric CG increased serum levels of IgG4 and in another Ig G4-related disease was reported., ${ }^{3,16}$

CG is a chronic disease, but there is still no guideline or effective treatment. ${ }^{13}$ There are different treatment strategies with limited efficacy including anti-secretory agents, PPI and H2-receptor antagonists, sucralfate, bismuth subsalycilate, corticosteroids, iron supplementation, hypoallergenic diets, and 5aminosalicylic acid. Only one study of 15 cases reported that there was no significant difference of clinical, endoscopic and histopathological outcome between PPI and dietary modifications such as cow's milk-free diet, gluten-free diet, and diet free of cow's milk, soy, egg, and wheat. ${ }^{3}$ Most of the patients had improvement of anemia with just oral iron supplements and PPI treatment. Iron deficiency recurrence has also been reported after treatment in different studies.,12 Improvement of the histological features of both CG and colitis was observed in only one male pediatric patient aged 11 years with adult-type disease who had been treated with oral prednisolone subsequently weaned onto long-term azathioprine treatment.? However this patient had additional histological changes in the duodenum on follow-up biopsy.

Childhood-onset CG is rare but should be included in the differential diagnosis in all children with intractable iron deficiency anemia. Endoscopy needs to be considered, even in an otherwise asymptomatic patient. Awareness must be heightened regarding the potentially increased long-term risk for autoimmune/ immune-related diseases. A specific therapy for collagenous colitis has not yet been established but oral iron supplementation and PPI is effective in treating the iron deficiency anemia 
in most patients. Careful long-term followup, including repeat endoscopies to monitor mucosal involvement is important. Further randomized clinical trials are needed.

\section{Author contribution}

The authors confirm contribution to the paper as follows: study conception and design: SÇ; data collection: SÇ, YÇ; analysis and interpretation of results: SÇ, YÇ; draft manuscript preparation: SÇ. All authors reviewed the results and approved the final version of the manuscript

\section{Conflict of interest}

The authors declare that there is no conflict of interest.

\section{REFERENCES}

1. Colletti RB, Trainer TD. Collagenous gastritis. Gastroenterology 1989; 97: 1552-1555. https://doi. org/10.1016/0016-5085(89)90403-4

2. Hijaz NM, Septer SS, Degaetano J, Attard TM. Clinical outcome of paediatric collagenous gastritis: case series and review of literature. World J Gastroenterol 2013; 19: 1478-1484. https://doi. org/10.3748/wjg.v19.i9.1478

3. Käppi T, Wanders A, Wolving, M, et al. Collagenous gastritis in children: incidence, disease course, and associations with autoimmunity and inflammatory markers. Clin Transl Gastroenterol 2020; 11: e00219. https://doi.org/10.14309/ctg.0000000000000219

4. Kamimura K, Kobayashi M, Sato Y, Aoyagi Y, Terai S. Collagenous gastritis: review. World J Gastrointest Endosc 2015; 7: 265-273. https://doi.org/10.4253/ wjge.v7.i3.265

5. Vesoulis Z, Lozanski G, Ravichandran P, Esper E. Collagenous gastritis: a case report, morphologic evaluation, and review. Mod Pathol 2000; 13: 591596. https://doi.org/10.1038/modpathol.3880101

6. Côté JF, Hankard GF, Faure C, et al. Collagenous gastritis revealed by severe anemia in a child. Hum Pathol 1998; 29: 883-886. https://doi.org/10.1016/ S0046-8177(98)90461-0
7. Matta J, Alex G, Cameron DJS, Chow CW, Hardikar W, Heine RG. Paediatric collagenous gastritis and colitis: a case series and review of the literature. J Pediatr Gastroenterol Nutr 2018; 67: 328-334. https:// doi.org/10.1097/MPG.0000000000001975

8. Ma C, Park JY, Montgomery EA, et al. A comparative clinicopathologic study of collagenous gastritis in children and adults: the same disorder with associated immune-mediated diseases. Am J Surg Pathol 2015; 39: 802-812. https://doi.org/10.1097/ PAS.0000000000000441

9. Suskind D, Wahbeh G, Murray K, Christie D, Kapur RP. Collagenous gastritis, a new spectrum of disease in paediatric patients: two case reports. Cases J 2009; 2: 7511. https://doi.org/10.4076/1757-1626-2-7511

10. Brain O, Rajaguru C, Warren B, Booth J, Travis S. Collagenous gastritis: reports and systematic review. Eur J Gastroenterol Hepatol 2009; 21: 1419-1424. https://doi.org/10.1097/MEG.0b013e32832770fa

11. Gopal P, McKenna BJ. The collagenous gastroenteritides: similarities and differences. Arch Pathol Lab Med 2010; 134: 1485-1489. https://doi. org/10.5858/2010-0295-CR.1

12. Lee YJ, Lee M, Kim D J, Lee S, Hong J. Three case reports of collagenous gastritis in children: Lessons for an endoscopic and histologic approach to mucosal nodularity of the stomach. Medicine (Baltimore) 2019; 98: e14870. https://doi.org/10.1097/ MD.0000000000014870

13. CamareroC, Leon F, ColinoE, etal.Collagenous colitis in children: clinicopathologic, microbiologic, and immunologic features. J Pediatr Gastroenterol Nutr 2003; 37: 508-513. https://doi.org/10.1097/00005176200310000-00020

14. Mandaliya R, Burkart AL, DiMarino AJ, Rattan S, Cohen S. Association between common variable immunodeficiency and collagenous infiltrative disorders of the gastrointestinal tract: a series of four patients. Indian J Gastroenterol 2016; 35: 133-138. https://doi.org/10.1007/s12664-016-0633-4

15. Anwar MS, Aamar A, Marhaba A, Sidhu JS Collagenous gastritis in a young female with $\operatorname{Ig} \mathrm{A}$ deficiency. Gastroenterology Res 2017; 10: 126-127. https://doi.org/10.14740/gr748w

16. Arnason T, Brown IS, Goldsmith JD, et al. Collagenous gastritis: a morphologic and immunohistochemical study of 40 patients. Mod Pathol 2015; 28: 533-544. https://doi.org/10.1038/modpathol.2014.119 